\title{
Molecular hydrogen traps within silicon
}

\author{
B. Hourahine ${ }^{\mathrm{a}, 1}$, R. Jones ${ }^{\mathrm{a}, 1}$, S. Öberg ${ }^{\mathrm{b}, 2}$, P. R. Briddon ${ }^{\mathrm{c}}$ \\ ${ }^{a}$ Department of Physics, The University of Exeter, Exeter, EX4 4QL, UK \\ b Department of Mathematics, University of Luleå, S95 187, Sweden \\ ${ }^{\mathrm{c}}$ Department of Physics, The University of Newcastle upon Tyne, \\ Newcastle upon Tyne, NE1 $7 R U, U K$
}

\begin{abstract}
We present the results of first principle calculations on the behaviour of molecular hydrogen within crystalline silicon, both as an isolated species, and within defects in the material. These results are compared with recent experimental infra-red and Raman data obtained for silicon treated by either hydrogen plasma or soaked in hydrogen gas. The effect of Fermi-level position on the diffusion barrier of molecular hydrogen within silicon is also discussed.
\end{abstract}

Interest in the behaviour of molecular hydrogen within silicon has been fuelled by the recent observation of $\mathrm{H}_{2}$ sited at three distinct environments within crystalline silicon by infrared $[1,2]$ and Raman $[3,4]$ spectroscopy.

\section{Hydrogen at $\mathrm{T}_{\mathrm{d} i}$}

Recent first principles calculations [5-7] find that $\left(\mathrm{H}_{2}\right)_{i}$ is sited at tetrahedral positions in the silicon lattice aligned along [100] or [111], and possessing a molecular stretch frequency directly comparable to the $3789 \mathrm{~cm}^{-1}$ mode observed by infrared and Raman spectroscopy $[2,4]$. The observation of a single IR and Raman active mode is consistent with the molecule being aligned along [110], and unable to rotate. If rotation were not hindered, then, for $\mathrm{T}_{\mathrm{d}}$ symmetry, a minimum of at least two IR lines would be expected in each of the $\mathrm{H}_{2}$, and $\mathrm{D}_{2}$ treated samples [8]. The [100] alignment can be also ruled out,

$\overline{1 \text { EPSRC }}$ and the ENDEASD network are thanked for computer time and financial support respectively.

2 Financially support by NFR and TFR, and computer support by PDC at KTH in Sweden are gratefully acknowledged.

Preprint submitted to Elsevier Preprint $\quad 30$ March 1999 
since only a single Raman active mode would be observed in this case, and no observable infrared signal. The [111] alignment would give a single Raman and IR active mode, but this alignment can be discounted since this line would split in HD samples.

\section{Lattice defects}

The low frequency of the interstitial molecule can be thought of as due to the leaking of charge from the surrounding bonds screening the intra-molecular proton-electron attraction [5]. If the size of the lattice cage were to be increased, for example, by a defect in the crystal, then the charge density at the centre of the cage due to the surrounding Si would fall. The molecular stretch frequency would then rise towards that of isolated hydrogen molecules.

Several types of extended defects in silicon are known to exist, with associated vibrational bands around $2100 \mathrm{~cm}^{-1}$, so the behaviour of $\mathrm{H}_{2}$ trapped at such sites becomes of interest in understanding the effects of plasma treatment on silicon.

\section{$2.1\langle 111\rangle$ platelets}

Of the models so far suggested for the $\langle 111\rangle$ platelet, TEM studies [9] and calculations [10], favour the structure with a pair of hydrogen atoms saturating each broken bond between (111) planes displaced apart by $\approx 3 \AA$. This structure does not include any silicon vacancies. Using the AIMPRO [11] code, the vibrational modes of a $\mathrm{H}_{2}$ molecule trapped within a platelet have been calculated. The stretch frequencies for the molecule lie at $4385 \mathrm{~cm}^{-1}$ for $\mathrm{H}_{2}$, 3796 and 3804 for $\mathrm{HD}$, and $3101 \mathrm{~cm}^{-1}$ for $\mathrm{D}_{2}$. The modes of the hydrogen saturating the surrounding silicon atoms fall between 2117 and $2098 \mathrm{~cm}^{-1}$. The experimental values for platelets $[12]$ lie at $\approx 2110$ and $\approx 1960 \mathrm{~cm}^{-1}$. The molecule has a bond length of $0.748 \AA$ when sited in the platelet, and is centred 2.459 (3.255) $\AA$ from the nearest passivating hydrogen (structural silicon) atoms.

\subsection{Voids}

An alternative structure at which molecules might be sited, would be a microvoid in the lattice $[13,14]$. Such voids are typically around $8 \mathrm{~nm}$ in diameter in crystalline silicon, this is somewhat large for ab initio modeling techniques, but 
some insight into the behaviour of hydrogen in such defects can be obtained by simulation of smaller structures. The smallest regular hydrogenated void that offers a more open environment than the crystal lattice is the passivated deca-vacancy. The vibrational frequencies of the molecule in such a void lie at $4324.6 \mathrm{~cm}^{-1}$ for $\mathrm{H}_{2}, 3745.9$ and 3743.8 for $\mathrm{HD}$, and 3057.9 for $\mathrm{D}_{2}$. The modes of the hydrogen passivating the void fall into three bands at 2205-2191, 21412129 and $2108-2099 \mathrm{~cm}^{-1}$ (experimentally [13] bands at $\approx 2125, \approx 2087$ and $\approx 2073 \mathrm{~cm}^{-1}$ are observed). The molecular bond length is $0.759 \AA$, and the distance of the centre of the structure to a $\mathrm{H}$ atom on the void surface is $3.36 \AA$.

\section{Molecular diffusion}

The weakening of the interstitial hydrogen molecule bond, due to screening effects from the surrounding lattice, might be expected to have an effect on the barrier to the molecule passing through a hexagonal site on its diffusion path through the crystal. If the charge density within the lattice was severely affected by excess carrier generated by, for example irradiation, then the diffusion barrier would be changed, and this is indeed what we find. The diffusion barrier drops from $0.73 \mathrm{eV}$ in the neutral case, to 0.33 and $0.46 \mathrm{eV}$ for clusters charged to +2 or -2 respectively, with an expansion of the bond length whilst the molecule is at the the hexagonal site from $0.788 \AA$ to 0.901 and $0.930 \AA$ respectively (The bond length at the tetrahedral site is insensitive to charge state).

\section{Conclusions}

The observation of a single IR and Raman active mode for $\mathrm{H}_{2}$ at a $\mathrm{T}_{\mathrm{d}}$ site is consistent with the isolated molecule being aligned along [110] and unable to rotate.

The calculated vibrational modes for $\mathrm{H}_{2}$ trapped at micro-voids in the crystal are close to the Raman line observed at $4158 \mathrm{~cm}^{-1}$ in samples exposed to hydrogen plasma $[3,4]$. This agreement would be expected to improve further if

anharmonic corrections to the frequency were included, which are $\approx 240 \mathrm{~cm}^{-1}$ for the free molecule [15]. The three calculated $\mathrm{Si}-\mathrm{H}$ stretch modes also agree reasonably well with the broad feature observed at $\approx 2100 \mathrm{~cm}^{-1}$. Similarly, the calculated molecular stretch frequency for $\mathrm{H}_{2}$ trapped at $\langle 111\rangle$ platelets of the type favoured by Deák et al also agree with the observed line, as are the calculated band of $\mathrm{Si}-\mathrm{H}$ stretch modes observed for the plasma treated material. 
The effect of Fermi-level position on the diffusion barrier and molecular bondlength of $\mathrm{H}_{2}$ in silicon may explain the observation that laser illumination causes a rapid decrease in the Raman signal intensity [16]. This observation can be explained as a result of the molecule becoming mobile even at cryogenic temperatures and travelling out of the observed region.

\section{References}

[1] R.E. Pritchard, M.J. Ashwin, J.H. Tucker, R.C. Newman, E.C. Lightowlers, M.J. Binns, S.A. Mcquaid, and R. Falster, Phys. Rev. B 56, 13118 (1997).

[2] R.E. Pritchard, M.J. Ashwin, J.H. Tucker, and R.C. Newman, Isolated interstitial hydrogen molecules in hydrogenated crystalline silicon, Phys. Rev. $\mathrm{B}$, (in press).

[3] K. Murakami, N. Fukata, S. Sasaki, K. Ishioka, M. Kitajima, S. Fujimura, J. Kikuchi, and H. Haneda, Phys. Rev. Lett. 77, 3161 (1996).

[4] A.W.R. Leitch and J. Weber (unpublished).

[5] C.G. Van de Walle, Phys. Rev. Lett. 80, 2177 (1998).

[6] Y. Okamoto, M. Saito, and A. Oshiyama, Phys. Rev. B 56, 10016 (1997).

[7] B. Hourahine, R. Jones, S. Öberg, R.C. Newman, P.R. Briddon, and E. Roduner, Phys. Rev. B 57, R12666 (1998).

[8] R. Jones and B. Hourahine (unpublished).

[9] S. Muto, S. Takeda, and M. Hirata, Phil. Mag. A 72, 1057 (1995).

[10] P. Deák, C.R. Ortiz, L.C. Snyder, and J.W. Corbett, Physica B 170, 223 (1991).

[11] R. Jones and P.R. Briddon, in Semiconductors and Semimetals, edited by M. Stavola (Academic Press, New York, in press), Chap. Identification of Defects in Semiconductors.

[12] N.M. Johnson, F.A. Ponce, R.A. Street, and R.J. Nemanich, Phys. Rev. B 35, 4166 (1987).

[13] H.J. Stein, S.M. Myers, and D.M. Follstaedt, J. Appl. Phys. 73, 2755 (1993).

[14] Y.J. Chabal and C.K.N. Patel, Phys. Rev. Lett. 53, 1771 (1984).

[15] K.P. Huber and G. Herzberg, Molecular Spectra and Molecular Structure IV (Van Nostrand Reinhold, New York, 1979).

[16] J. Weber, Private communication. 